Proceedings of the $2^{\text {nd }}$ ICEENG Conference, 23-25 Nov. 1999

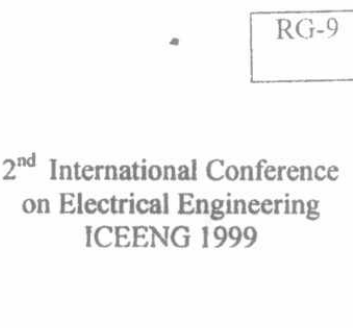

Military Technical College Kobry Elkobbah,

Cairo, Egypt

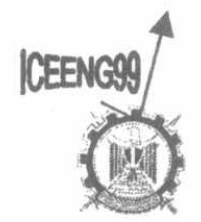

$2^{\text {nd }}$ International Conference

Electrical Engineerin

ICEENG 1999

\title{
AN ALGORITHM FOR CALCULATING THE AERODYNAMIC DERIVATIVES OF A SUPER-SONIC MISSILE
}

\author{
K.G.Aly*, and G.A.El-Sheikh**
}

\begin{abstract}
Determination of the aerodynamic derivatives is considered as an essential procedure when analyzing the flight path trajectory. This trajectory is determined by the aerodynamic forces and moments; which are functions of these derivatives. So, as accurately these derivatives are calculated as accurately the flight path trajectory will be determined. Thus, calculation of the aerodynamic derivatives is necessary for a six-degree-of-freedom missile trajectory simulation. Aerodynamics is part of the missile's airframe subsystem, the other major parts being propulsion and structure. The aerodynamics is closely related to the autopilot and controls that, in turn, form a part of the overall guidance loop. To evaluate the guidance, control, and autopilot behavior, it is required to achieve the three-dimensional representation of the aerodynamic forces and moments coefficients. The intent of the three dimensional representation of aerodynamics is to be able to analyze the missile performance throughout its potential operational regime and not just in the neighborhood of the trim points. The sixdegree-of-freedom trajectory simulation, using these three dimensional data, can be used to predict missile performance against maneuvering targets and to troubleshoot flight problems by reconstructing the flight trajectory from on-board measurements of missile parameters.

This paper presents an algorithm for calculating the aerodynamic derivatives of a supersonic missile. This algorithm depends on many parameters such as missile aerodynamic configuration shape and dimensions, atmospheric data, positions of center of gravity and center of pressure, angle of attack, side slip angle, control surfaces deflections, and a set of the aerodynamic based data and NASA curves. The atmospheric data are the air density, pressure of the air, the air viscosity and speed of sound. A mathematical model is provided. Software package in the MATLAB environment is developed to calculate the aerodynamic coefficients. The intent from determination of these coefficients is to calculate the aerodynamic forces and moments that affect on the missile during its flight in order to determine the missile trajectory and evaluate the missile control and guidance systems. The effect of the aerodynamic derivatives on the flight path trajectory is analyzed. This effect is discussed in two cases: the simplified case and the complete case. In the simplified case these derivatives are calculated in the supersonic speeds and estimated in the subsonic and transonic ranges. In the complete case these derivatives are calculated in the subsonic, transonic and supersonic speeds. The results show that the complete case is the better case from the viewpoint of the flight parameters (smallest miss distance and smallest normal acceleration).
\end{abstract}

Keywords: Aerodynamics, Guidance and Control, and Mechanics of flight.

* Ph.D. student, Syrian Army

** Dr. , Radar and Guidance Dpt., Military Technical College, Cairo, Egypt 
Proceedings of the $2^{\text {nd }}$ ICEENG Conference, 23-25 Nov. 1999

\begin{tabular}{|l|l|}
\hline $\mathrm{RG}-9$ & 2 \\
\hline
\end{tabular}

\section{1-INTRODUCTION}

Determination of the aerodynamic derivatives is considered as an essential procedure when analyzing the flight path trajectory. This trajectory is determined by the aerodynamic forces and moments; which are functions of these derivatives. So, as accurately these derivatives are calculated as accurately the flight path trajectory is determined. Thus, calculation of the aerodynamic derivatives is necessary for a six-degree-of-freedom missile trajectory simulation. Aerodynamics is part of the missile's airframe subsystem, the other major parts being propulsion and structure. The aerodynamics is closely related to the autopilot and controls that, in turn, form a part of the overall guidance loop. The evaluation or enhancement of the guidance, control, and autopilot behavior necessitates a three-dimensional representation of the aerodynamic forces and moments coefficients. The intent of this representation is to be able to analyze the missile performance throughout its potential operational regime and not just in the neighborhood of the trim points. The six-degree-offreedom trajectory simulation, using these three dimensional data, can be used to predict missile performance against maneuvering targets and to troubleshoot flight problems by reconstructing the flight trajectory from on-board measurements of missile parameters [1]. The forces and moments acting on the missile depend on a set of the aerodynamic coefficients. The coefficients of the aerodynamic forces are $c_{x}, c_{y}$, and $c_{z}$ while the coefficients of the aerodynamic moments are $m_{x}, m_{y}$, and $m_{z}$. The wind tunnel tests are considered as the accurate estimation of these coefficients. However for the theoretical estimation, the aerodynamic coefficients are obtained by an interpolation of an aerodynamic database that is provided by the aerodynamic and NASA reports [2]. The aerodynamic derivatives are calculated for the body and each of the aerodynamic surfaces, together with estimates for the interference effects of the various components on each other and for different mach numbers. The aerodynamic coefficients do not depend only on mach number, but also on the missile incidence and deflection angles for the control surfaces [3].

In this paper, the aerodynamic description of the missile configuration over full operating range of altitude, mach number, Reynolds number, angles of attack and sideslip, and control surfaces deflections in pitch, yaw, and roll is provided depending on the data presented in NASA and aerodynamic reports. In addition, the center of gravity, center of pressure positions, and the moments of inertia of the missile around its axes are determined [4]. The aerodynamic performance of the missile is investigated during the whole time of flight. The underlying missile is a command-guided system, which consists of a ground-based guidance radar that determines the coordinates of the engaged air target and the pursuing missile. The guidance commands are proportional to the lateral displacement of the missile from the target line of sight [5]. This missile is aerodynamically controlled via tail control fins and has two planes of symmetry. So, it is enough to calculate the aerodynamic derivatives in the pitch plane only. A mathematical model is developed to describe the system under consideration. The characteristic dimensions for the underlying missile are shown in Fig. 1.

\section{2-AN ALGORITHM FOR CALCULATING THE AERODYNAMIC DERIVATIVES}

The aerodynamic derivatives depend on many parameters such as missile aerodynamic configuration shape, atmospheric data, flight parameters as mach number, incidence angles 
and control surfaces deflections. A flow chart for the determination of the aerodynamic derivatives is shown in Fig. 2. According to the missile aerodynamic shape, the necessary missile configuration data and the geometric characteristics are determined. Also, variations of the atmospheric data (speed of sound, viscosity of air, air density) with the altitude are to be determined within the missile altitude operating range. A set of the NASA aerodynamic curves and data are used for the aerodynamic descriptions. Using all of these data, there are two approaches for calculating the aerodynamic derivatives: either to concentrate on the flight duration of guidance only or to consider the whole envelope of flight. The two approaches are considered in the next subsections and evaluated through trajectories analysis later in this paper.

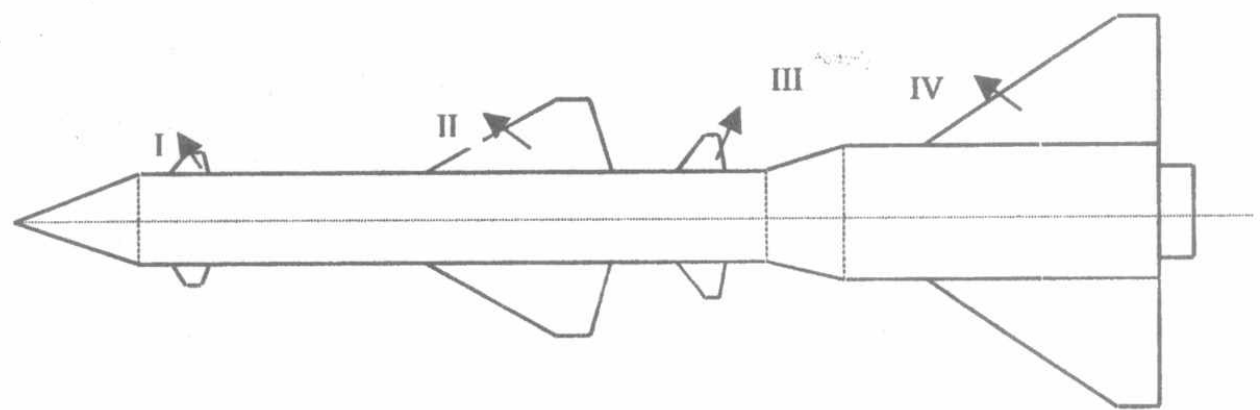

Fig. 1: The hypothetical missile configuration

\section{2-1 Simplified Approach For Calculating The Aerodynamic Derivatives}

This approach depends on a set of aerodynamic based data, atmospheric data, and missile aerodynamic configuration shape and flight characteristics. These data are used to calculate the aerodynamic derivatives for forces and moments acting on the missile during the guided phase of its flight.

\subsubsection{Lift force coefficients}

The total lift force coefficient $c_{y 1}$ is composed of two main components as follows [1]:

$c_{y 1}=c_{y_{1}}^{\alpha} \alpha+c_{y_{1}}^{\delta} \delta$

The total lift force derivative $c_{y_{1}}^{\alpha}$ is given by the relation [6]:

$c_{y_{1}}^{\alpha}=c_{y_{f}}^{\alpha}+c_{y_{w(f)}}^{\alpha}+c_{y_{f(w)}}^{\alpha}+c_{y_{t(f)}}^{\alpha}+c_{y_{f(t)}^{\alpha}}^{\alpha}+c_{y_{t(w)}}^{\alpha}$

Where $c_{y_{f}}^{\alpha}$ is the derivative of a single body,

$c_{y_{w(f)}^{\alpha}}^{\alpha}$ is the derivative of the wing in the presence of the body,

$c_{y_{f(w)}}^{\alpha}$ is the derivative of the body in the presence of the wing,

$c_{y_{t(f)}^{\alpha}}^{\alpha}$ is the derivative of the tail wing in the presence of the body,

$c_{y_{f(t)}^{\alpha}}^{\alpha}$ is the derivative of the body and the tail wings,

$c_{y_{t(w)}}^{\alpha}$ is the derivative of the interference between the tail wings and the wings, 


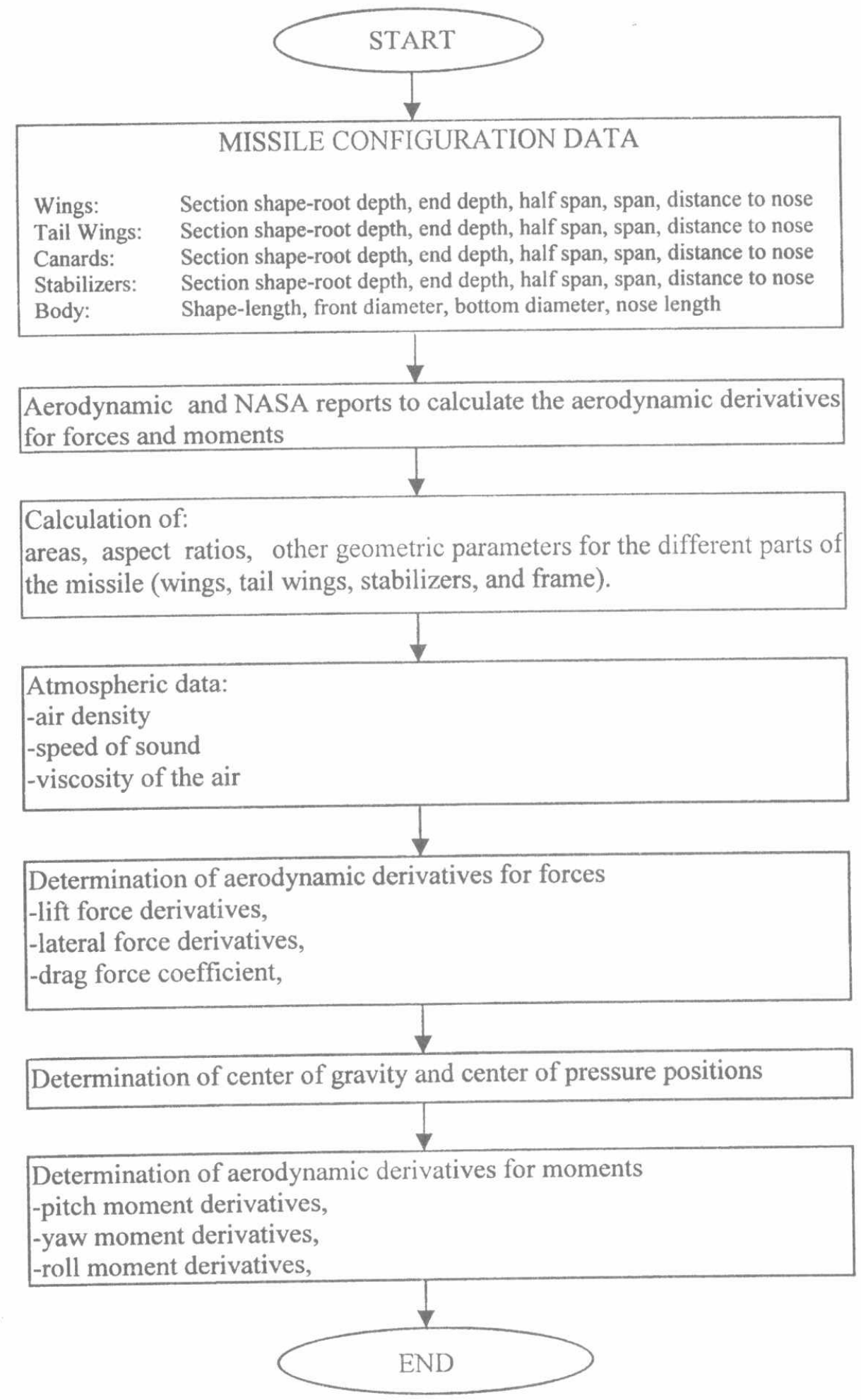

Fig. 2: Flow chart for determination of the aerodynamic derivatives 
The total lift force derivative $c_{y_{1}}^{\delta}$ is given by the relation [6]:

$c_{y_{1}}^{\delta}=c_{y_{t(f)}}^{\delta}+c_{y_{f(t)}}^{\delta}$

Where $c_{y_{t(f)}}^{\delta}$ is the lift force derivative of the tail fins in the presence of the body,

$c_{y_{f}(t)}^{\delta}$ is the lift force derivative of the body in the presence of the tail fins.

Variations of the lift force derivatives $c_{y_{1}}^{\alpha}$ and $c_{y_{1}}^{\delta}$ against mach number are shown in Fig. 3.
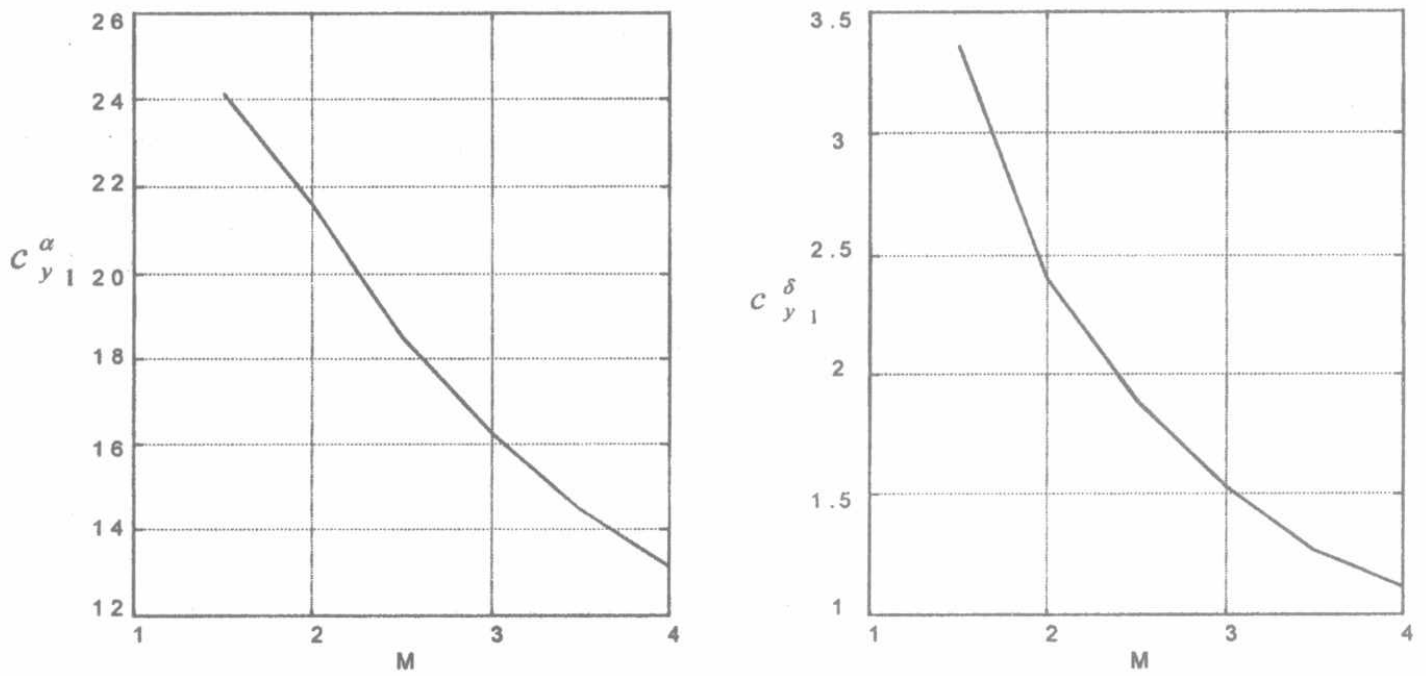

Fig. 3: Lift force derivatives $c_{y_{1}}^{\alpha}$ and $c_{y_{1}}^{\delta}$ versus mach number for the simplified apparoach

It is clear that as mach number increases both the $c_{y_{1}}^{\alpha}$ and $c_{y_{1}}^{\delta}$ decreases.

\subsubsection{Drag force coefficient}

The total drag coefficient of the missile is given by [1]:

$c_{x}=c_{x_{o}}+c_{x_{i}}$

Where $c_{x o}$ is the missile coefficient of drag at zero lift, it is given by [6]:

$c_{x_{o}}=K_{S N}\left[c_{x_{o f}} \frac{s_{f}}{s}+c_{x_{o w}} \frac{A_{w}}{s}+c_{x_{o t}} \frac{A_{f}}{s}\right]$

$K_{S N}$ is the correction factor and it is taken $1.3[6], c_{x_{\text {of }}}$ is the body zero lift drag coefficient of the body, $c_{x_{o w}}$ is the zero lift drag coefficient of the wings, $c_{x_{o t}}$ is the zero lift drag coefficient of the tail wings, $s_{f}$ is the area of body cross-section, $A_{w}$ is the wing area, $A_{t}$ is the tail wing area, $s$ is the reference area, generally it is taken as the body cross-section area. For small $\alpha$ the coefficient of induced drag can be approximately evaluated as [6]: 
Proceedings of the $2^{\text {nd }}$ ICEENG Conference, 23-25 Nov. 1999

\begin{tabular}{|l|l|}
\hline$R(i-9)$ & 6 \\
\hline
\end{tabular}

$$
c_{x_{i}}=c_{y_{1}} \sin (\alpha)
$$

\subsubsection{Aerodynamic moment coefficients}

The total aerodynamic moment coefficients $m_{z 1}$ is given by the equation [1]:

$m_{z 1}=m_{z 1}^{\alpha} \alpha+m_{z 1}^{\delta} \delta+m_{z}^{\omega_{z}} \omega_{z}$

To evaluate the position of neutral point of individual parts of the missile $x_{f_{i}}$, we evaluate derivative of moment with respect to the nose of the missile $\left(m_{z_{1}}^{\alpha}\right)_{\text {nose }}$ and this will be recalculated to the derivative $\left(m_{z 1}^{\alpha}\right)_{c . g}$. related to the center of gravity. The total moment coefficient $\left(m_{z 1}^{\alpha}\right)_{\text {nose }}$ is given by the relation [6]:

$\left(m_{Z_{1}}^{\alpha}\right)_{n o s e}=m_{Z_{f}}^{\alpha}+m_{Z w(f)}^{\alpha}+m_{Z f(w)}^{\alpha}+m_{Z_{t}(f)}^{\alpha}+m_{Z_{f(t)}}^{\alpha}+m_{Z_{t(w)}}^{\alpha}$

The zero lift drag force coefficient $C_{x o}$ and the moment derivative $m_{z_{1}}^{\alpha}$ against mach number are shown in Fig . 4.
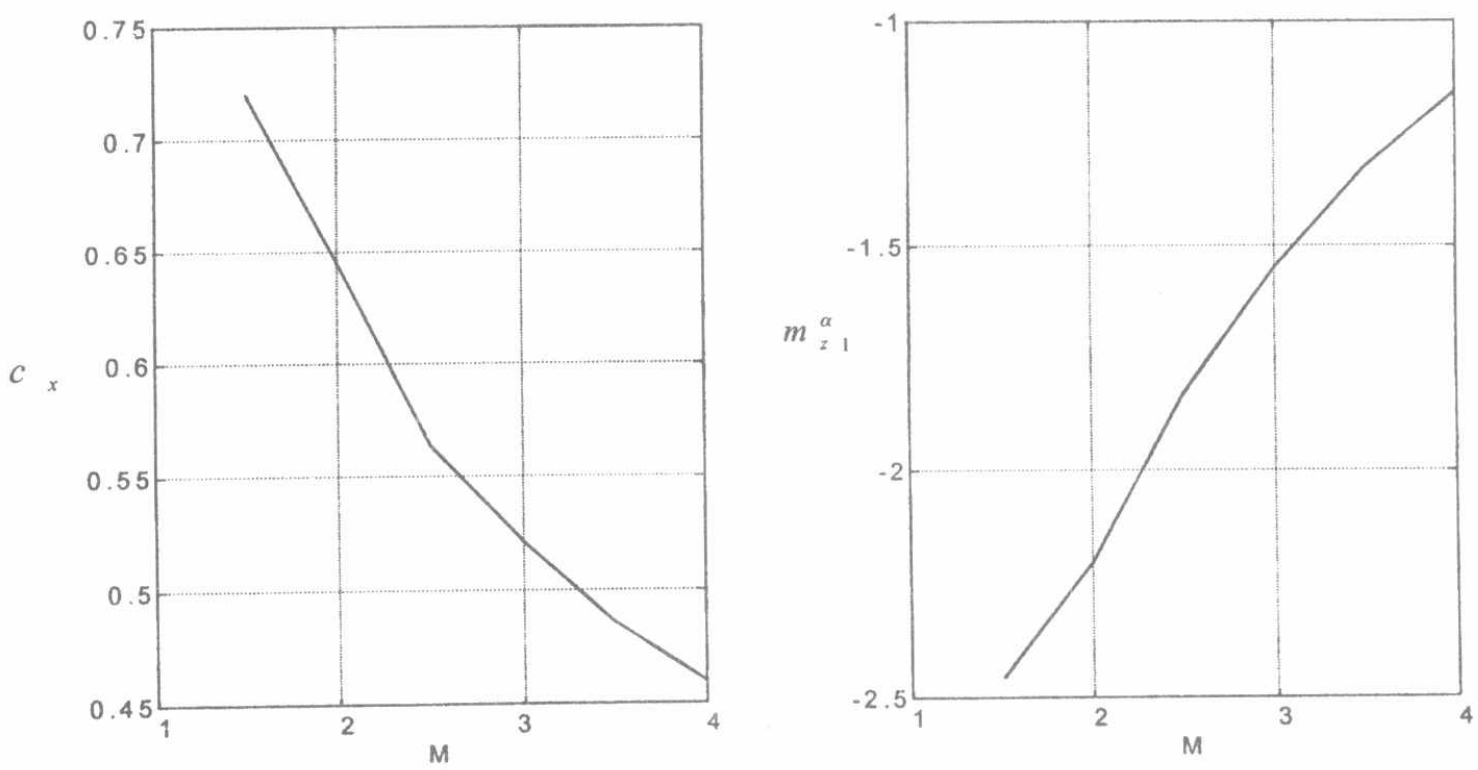

Fig. 4: Zero lift force coefficient $c_{x o}$ and moment derivative $m_{z 1}^{\alpha}$ versus mach number for the simplified approach

The figure shows that as the mach number increases the missile zero lift drag coefficient decreases. Also, the figure shows that $m_{=1}^{\alpha}$ is negative, so the missile is statically stable. It decreases in the absolute value as the mach number increases.

When evaluating $m_{z 1}^{\delta}$ we are considering the point of application of the lift caused by a deflection of the tail wings as identical to the point of application of the lift caused by an angle of attack change, it is given by the relation [6]: 
$m_{z 1}^{\delta}=m_{z t(f)}^{\delta}+m_{z f(t)}^{\delta}$

The damping pitching moment $m_{z}^{\omega_{z}}$ is given by the relation [6]:

$m_{z}^{w_{z}}=m_{z f}^{w_{z}}+m_{z}^{w_{z}} w_{(f)}+m_{z f(w)}^{w_{z}}+m_{z}^{w_{z}}{ }_{t(f)}+m_{z f(t)}^{w_{z}}+m_{z t(w)}^{w_{z}}$

Variations of the moment derivative $m_{z 1}^{\delta}$ and the damping moment derivative $m_{z}^{\omega_{z}}$ against mach number are shown in Fig. 5.
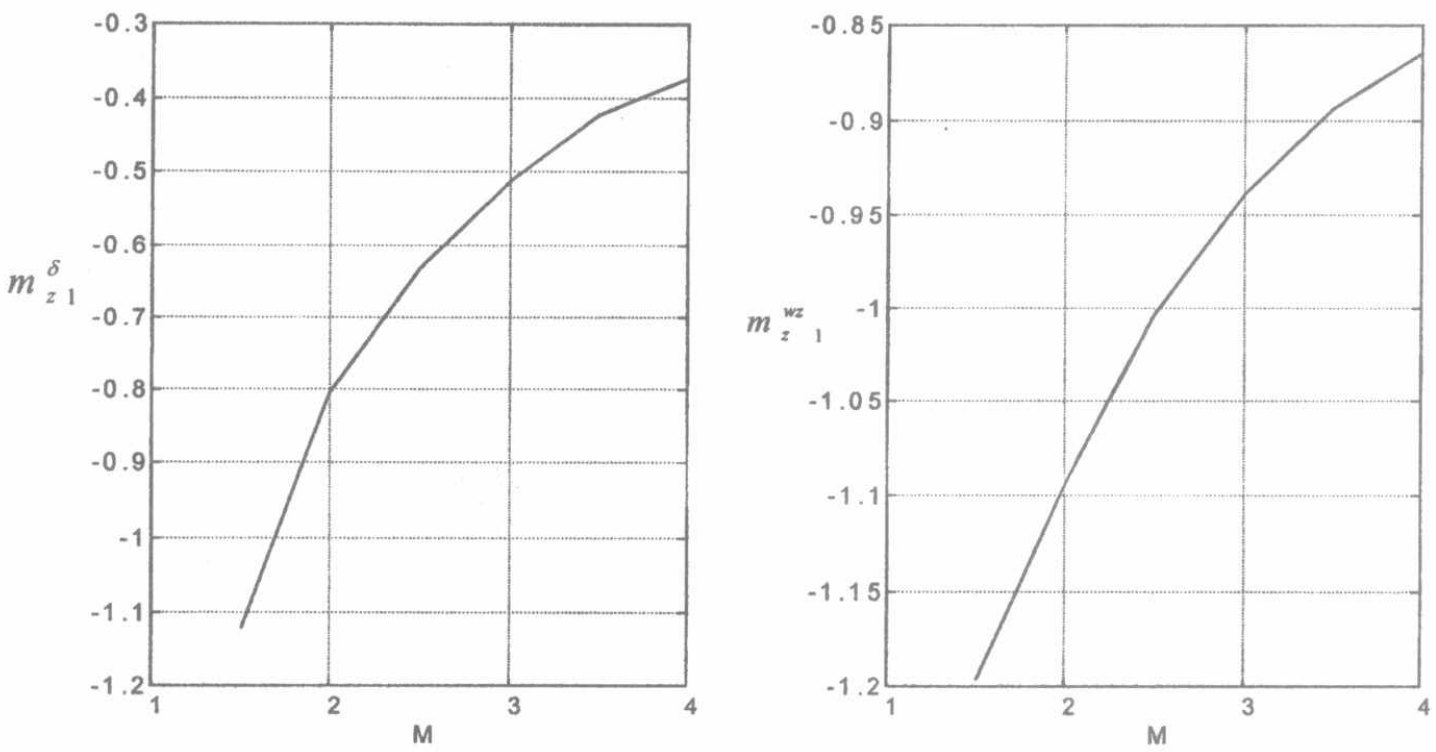

Fig. 5: Moment derivative $m_{z 1}^{\delta}$ and daming moment derivative $m_{z}^{\omega_{z}}$ versus mach number for the simplified approach

The figure shows that $m_{z 1}^{\delta}$ is negative and decreases in the absolute value as the mach number increases. Also, the figure shows that $m_{z}^{\omega_{z}}$ is negative and it decreases in the absolute value as the mach number increases.

\section{2-2 Complete Approach For Calculating The Aerodynamic Derivatives}

Similarly this approach depends on a set of aerodynamic based data, atmospheric data, missile aerodynamic configuration shape and flight characteristics. However, these data are used to calculate the aerodynamic derivatives for forces and moments allover the envelope of missile flight.

\subsubsection{Lift force coefficients}

The total lift force coefficient $c_{y_{1}}$ is composed of two main components as given by the equation (1). The lift force derivative $c_{y_{1}}^{\alpha}$ is determined by [7]: 
$c_{y_{1}}^{\alpha}=\left(c_{y_{1}}^{\alpha} \bar{s}\right)_{b}+\sum_{k=I}^{I V}\left(c_{y_{1}}^{\alpha} \bar{s} k_{T}\right)_{k}$

Where $\left(c_{y_{1}}^{\alpha}\right)_{b}$ is the derivative of isolated body, $(\bar{s})_{b}=\frac{s_{b}}{s_{f}},(\bar{s})_{k}=\frac{s_{k}}{s_{f}}$

$s_{b}$ is the body cross section area, and $s_{f}$ is the reference area (cross section area),

$s_{k}$ is the area of surface number k, where k=I, II, III, IV as depicted in Fig. 1.

$\left(c_{y_{1}}^{\alpha}\right)_{k}$ is the lift force derivative of surface number $\mathrm{k}$,

$\left(K_{T}\right)_{k}$ is the air pressure ratio at the surface number $\mathrm{k}$ and at the missile nose.

The total lift force derivative $c_{y_{1}}^{\delta}$ is given by [8]:

$c_{y_{1}}^{\delta}=\left(c_{y_{1}}^{\delta} \bar{s} k_{T}\right)_{\text {tail }}$

Where $\left(c_{y_{1}}^{\delta}\right)_{\text {tail }}$ is the lift force derivative due to the rear control surfaces. Variations of the lift force derivatives $c_{y_{1}}^{\alpha}$ and $c_{y_{1}}^{\delta}$ against mach number are shown in Fig. 6.
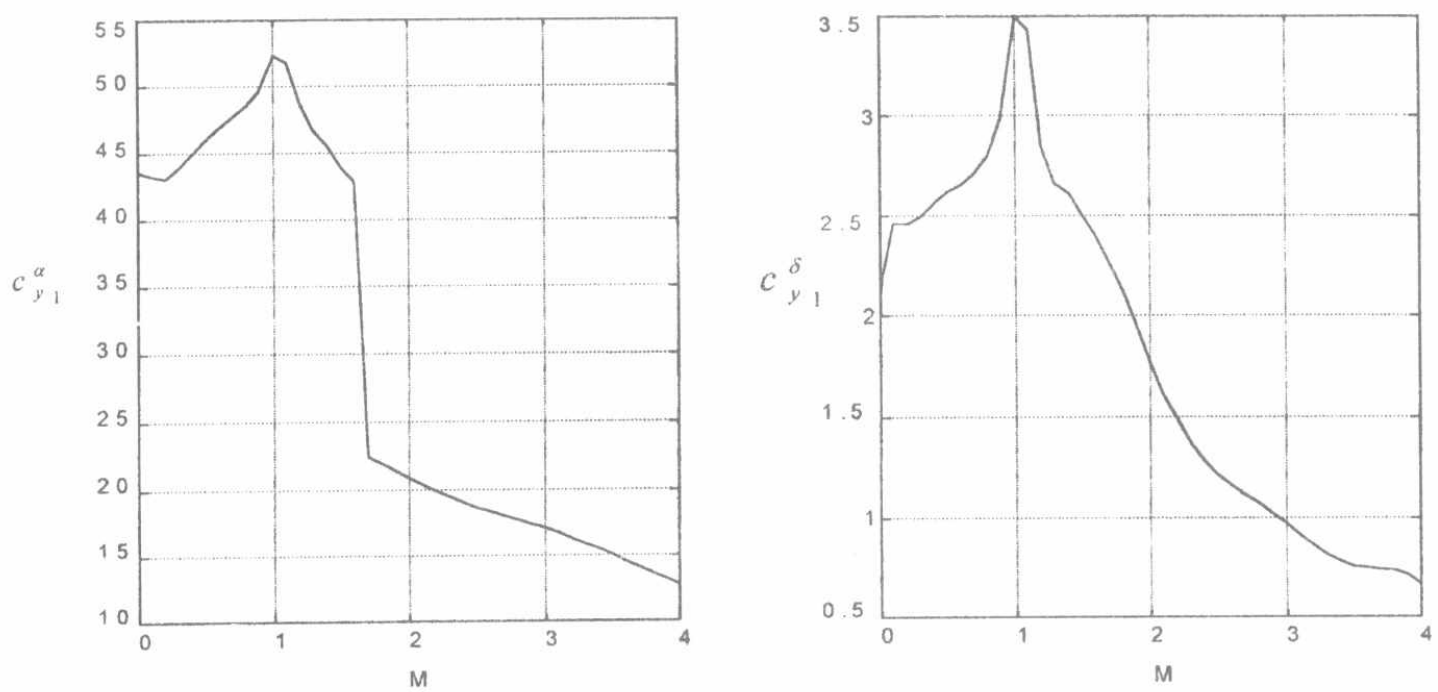

Fig. 6: Lift force derivatives $c_{y_{1}}^{\alpha}$ and $c_{y 1}^{\delta}$ against mach number for the complete approach

The figure shows that $c_{y_{1}}^{\alpha}$ in the first stage of flight is higher than in the second stage of flight due to the lift from the stabilizers. In the subsonic range the lift contribution of the body and the mentioned surfaces increases as the mach number increases; this verifies that we need more lift force for the missile in this stage, but in the supersonic range as the mach number increases the lift contribution of the body and surfaces decreases. Also, as shown that $c_{y 1}^{\delta}$ in the first stage increases as the mach number increases. In the second stage it decreases as the mach number increases. The lateral force derivatives are given by the following relations [7]:

$c_{z 1}^{\beta}=-c_{y 1}^{\alpha}$ 
$c_{z 1}^{\delta}=-c_{y 1}^{\delta}$

\subsubsection{Drag force coefficient}

The missile drag force coefficient $c_{x}$ is given by [1]:

$$
c_{x}=c_{x o}+c_{x i}
$$

Where $c_{x_{o}}$ is total zero lift drag force coefficient (when there is no incidence or deflection angles) and it is given by [7]:

$c_{x_{o}}=k_{s}\left[\left(c_{x_{o}} \bar{s}\right)_{b}+\left(c_{x_{o}} \bar{s}\right)_{b 1}+\left(c_{x_{o}} k_{T} \sum \bar{s}\right)_{k}\right]$

Where $k_{s}$ is the correction factor and it is taken 1.05 [8],

$(\bar{s})_{b 1}$ is the cross section area of the first stage body related to reference area,

$\left(\sum \vec{s}\right)_{k}$ is the total areas of the surfaces number $\mathrm{k}$ related to reference area,

$\left(c_{x_{o}}\right)_{b}$ is the drag force coefficient of the second stage body, it is given by [7]:

$\left(c_{x o}\right)_{b}=\left(c_{x f}+c_{x p}\right)_{b}$

Where $\left(c_{x_{f}}\right)_{b}$ is the body skin friction coefficient, $\left(c_{x p}\right)_{b}$ is the body pressure drag coefficient, $\left(c_{x_{o}}\right)_{b 1}$ is the drag force coefficient of the first stage body, $\left(c_{x_{o}}\right)_{k}$ is the drag force coefficient of the surface $\mathrm{k}$. It is given by [7]:

$\left(c_{x_{o}}\right)_{k}=\left(c_{x_{f}}+c_{x_{w}}\right)_{k}$

Where $\left(c_{x_{f}}\right)_{k}$ is the friction coefficient of the surface number $\mathrm{k}$, $\left(c_{x_{w}}\right)_{k}$ is the wave drag coefficient of the surface number $\mathrm{k}$,

\subsubsection{Aerodynamic moment coefficients}

The pitching moment derivative $m_{z 1}^{\alpha}$ is the main pitching moment derivative and given by the equation [7]:

$m_{z 1}^{\alpha}=c_{y_{1}}^{\alpha} \frac{x_{c g}-x_{F_{a}}}{L}$

Where $L$ is the reference length and it is taken as the missile length, $x_{c g}$ is the center of gravity position, and $x_{F_{\alpha}}$ is the center of pressure position.

The drag force coefficient $c_{x}$ and the pitching moment derivative $m_{z 1}^{\alpha}$ against mach number are shown in Fig. 7. 

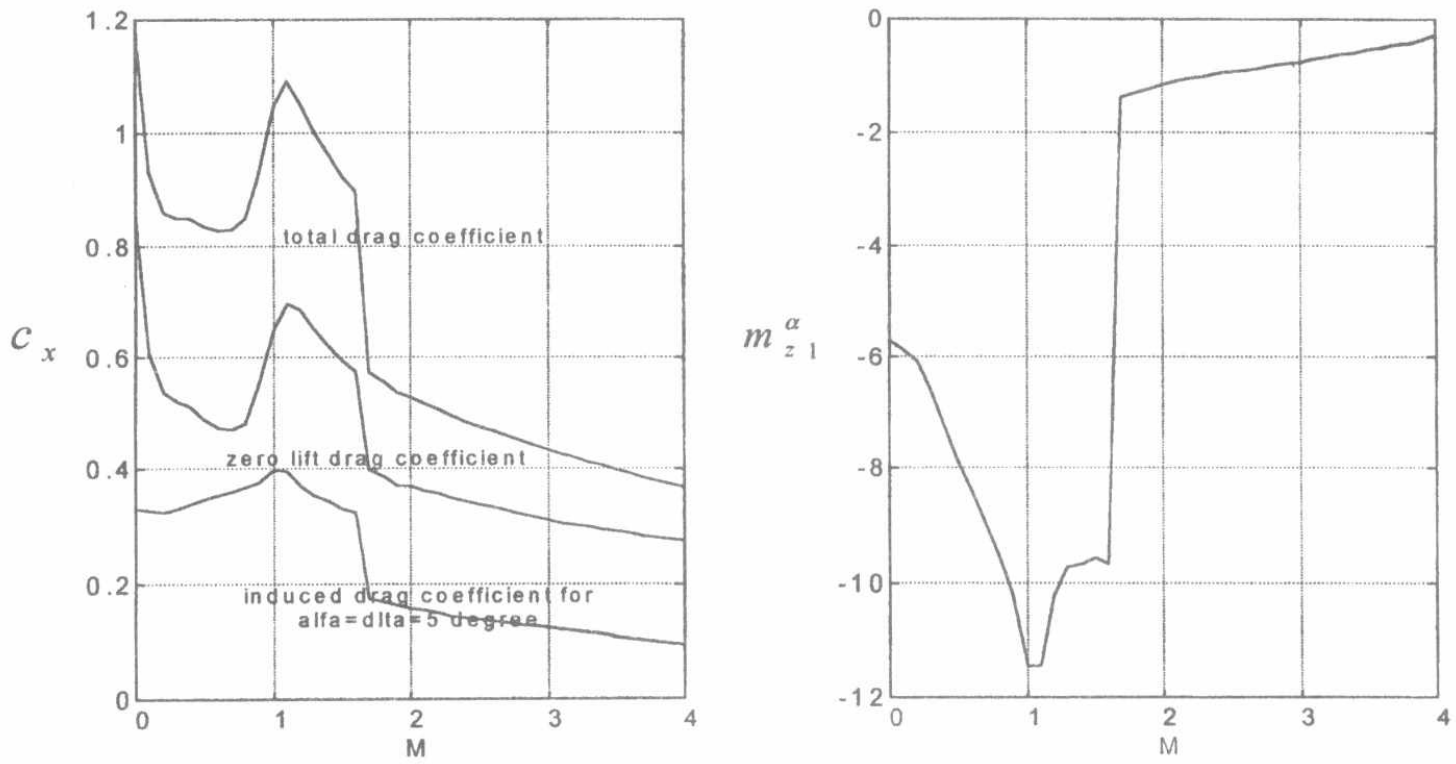

Fig. 7: Drag force cofficent $c_{x}$ and pitching moment derivative $m_{z 1}^{\alpha}$ against mach number for the complete approach

The figure shows that the induced drag force coefficient decreases as the mach number increases, the zero lift drag coefficient decreases as the mach number increases in the subsonic and supersonic ranges but it increases as the mach number increases in the transonic range for the underlying missile configuration. In the transonic range, as the nose drag coefficient increases sharply, the total zero lift drag coefficient increases as the mach number increases in spite of the decreases of the skin friction drag. Also, the figure shows that $m_{z 1}^{\alpha}$ is negative, so the missile is statically stable. In the first stage it is higher than in the second stage due to the pitching moment derivative from the stabilizers surfaces. In the subsonic range as the mach number increases $m_{z 1}^{\alpha}$ increases in the absolute value, but in the supersonic range it decreases by the increase of the mach number in the absolute value.

The total pitching moment derivative $m_{z 1}^{\delta}$ is given by the equation [7]:

$m_{z 1}^{\delta}=\left(m_{z 1}^{\delta}\right)_{\text {tall }}=\left(c_{y_{1}}^{\delta}\right)_{\text {tail }} \frac{x_{c g}-\left(x_{F_{\delta}}\right)_{\text {tail }}}{L}$

The pitching moment derivative $m_{z}^{w z}$ is called the damping pitching moment derivative, it arises due to the pitching rate of the missile and given by the following relation [7]:

$m_{z=1}^{w z}=\left(m_{z}^{w z} \bar{s}^{2}\right)_{b}+\sum_{k=1}^{I V}\left(m_{z}^{w z}, \bar{s}^{2}{ }^{2} \sqrt{k_{T}}\right)_{k}$

Where $\left(m_{z_{1}}^{a x}\right)_{b}$ is the pitching moment derivative of the body, $\left(b_{A k}\right)_{k}$ is the aerodynamic chord of the surface $\mathrm{k},\left(m_{z}^{\omega z}\right)_{k}$ is the pitching moment derivative of the surface number $\mathrm{k}$.

The pitching moment derivative $m_{z 1}^{\delta}$ and $m_{z, 1}^{w z}$ are shown in Fig. 8. 

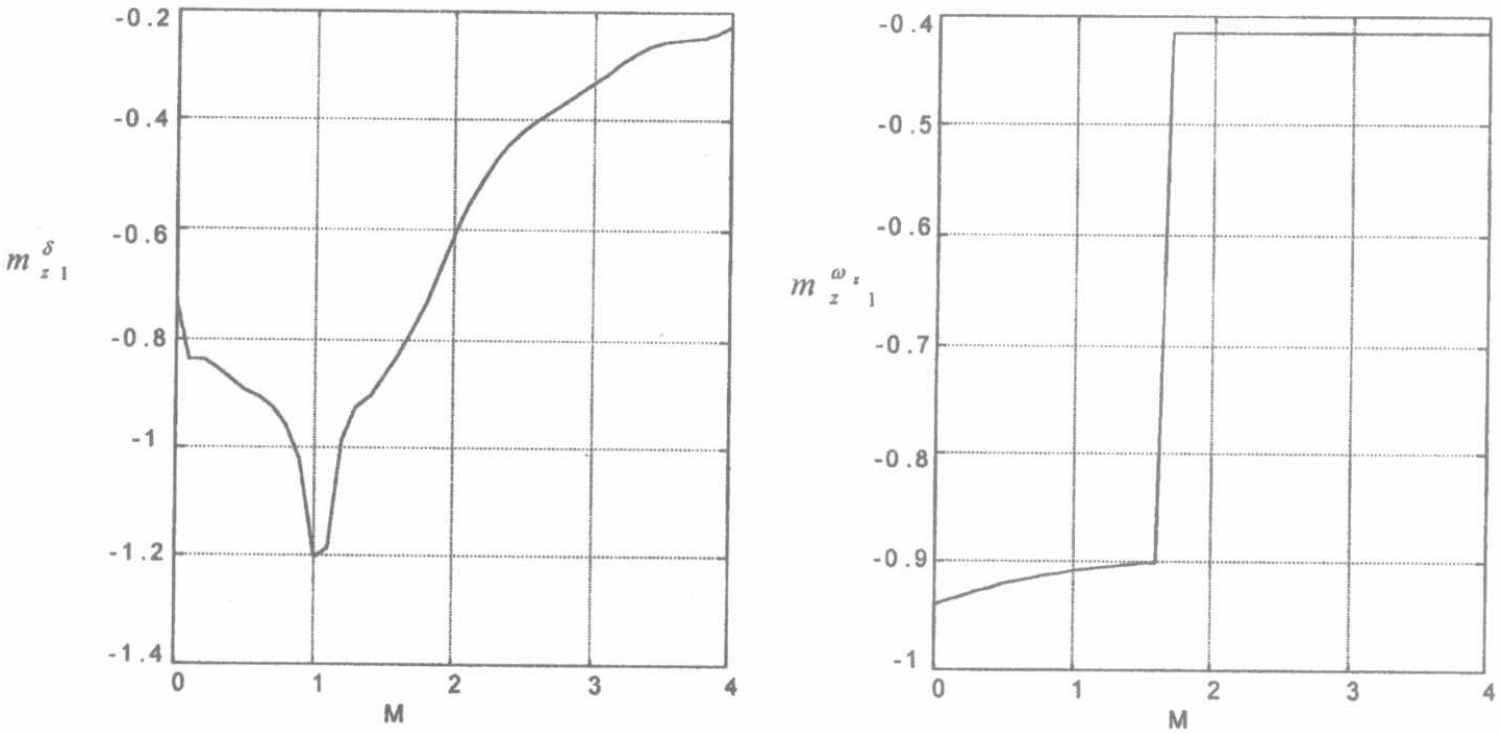

Fig. 8: Pitching moment derivative $m_{z 1}^{\delta}$ and $m_{z 1}^{\omega_{z}}$ against mach number for complete approach

The figure shows that $m_{z 1}^{\delta}$ is negative due to using rear control surfaces. In the subsonic range it increases as the mach number increases. In the supersonic range it decreases as the mach number increases. Also, the figure shows that $m_{z 1}^{\omega_{z}}$ is negative, its values in the first stage are higher than in the second stage and it decreases as the mach number increases in the absolute value. The lateral moment derivatives are given by [7]:

$m_{y_{1}}^{\beta}=m_{z 1}^{\alpha}\left(\frac{L}{l_{w}}\right)$

$m_{y_{1}}^{\delta}=m_{z 1}^{\delta}\left(\frac{L}{l_{w}}\right)$

$m_{y}^{w y}=2\left(\frac{L}{l_{w}}\right) m_{z}^{w z}$

Where $l_{w}$ is the wing tip to tip distance and it is considered as the reference length for the lateral moments [8].

\section{3-Flight Path Analysis}

The missile moves in space under the effect of gravity, thrust, and aerodynamic forces. The aerodynamic forces and moments that affect the missile may be expressed by the following equations [9]:

$F_{x}=-c_{x} s q$

$F_{y}=c_{y} s q$

$F_{z}=-c_{z} s q$ 


$$
\begin{aligned}
& M_{x}=m_{x} s q_{x} \\
& M_{y}=m_{y} s q l_{y} \\
& M_{z}=m_{z} s q l_{z}
\end{aligned}
$$

Where $F_{x}, F_{y}, F_{z}, M_{x}, M_{y}$ and $M_{z}$ are the aerodynamic forces and moments,

$l_{x}, l_{y}$ and $l_{z}$ are the characteristic linear dimensions of the missile,

and $q$ is the dynamic pressure.

Since the forces acting upon the missile are not in the same directions, so different coordinate systems are adopted. Ground coordinate system, velocity coordinate system, and body coordinate system are mostly used. Usually, the aerodynamic forces and missile velocity are analyzed in the velocity coordinate system, the thrust force is analyzed in the body coordinate system, and the gravity force is analyzed in the ground coordinate system. For correct study and analysis of the guided missile motion, suitable transformations should be achieved between the coordinate systems constituting part of systems simulation. Simulation is a process through which, the effect of various parameters on the guided missile system behavior can be estimated. Toward this simulation a 6-degrees of freedom model is adopted including different modules, airframe, autopilot, guidance, kinematics, and missile-target geometry. Each module represents a physically existing system as shown in Fig. 9.

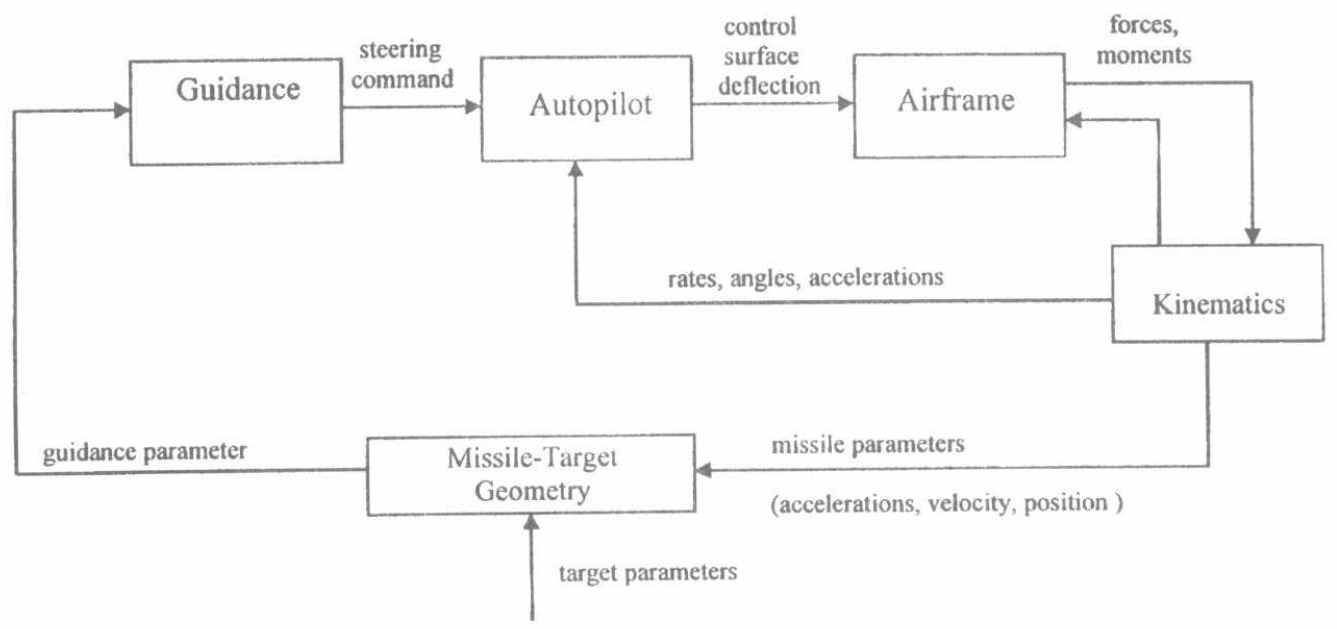

Fig. 9: Missile trajectory simulation block diagram

According to this block diagram, the relative coordinates between target and missile are determined for which guidance parameters are calculated and applied to the module of command guidance generation. This module produces the guidance commands used to steer the missile in space. These commands are proportional to the demanded acceleration and are applied through the autopilot to the airframe module. This module is responsible of fin deflections and consequently the forces and moments acting on the missile. Due to these forces and moments, the flight parameters change and consequently the kinematics module provides measured values for acceleration, velocity, position, angles, rates, and angular accelerations. This scenario of missile flight simulation is described in algorithmic form as shown in Fig. 10. 


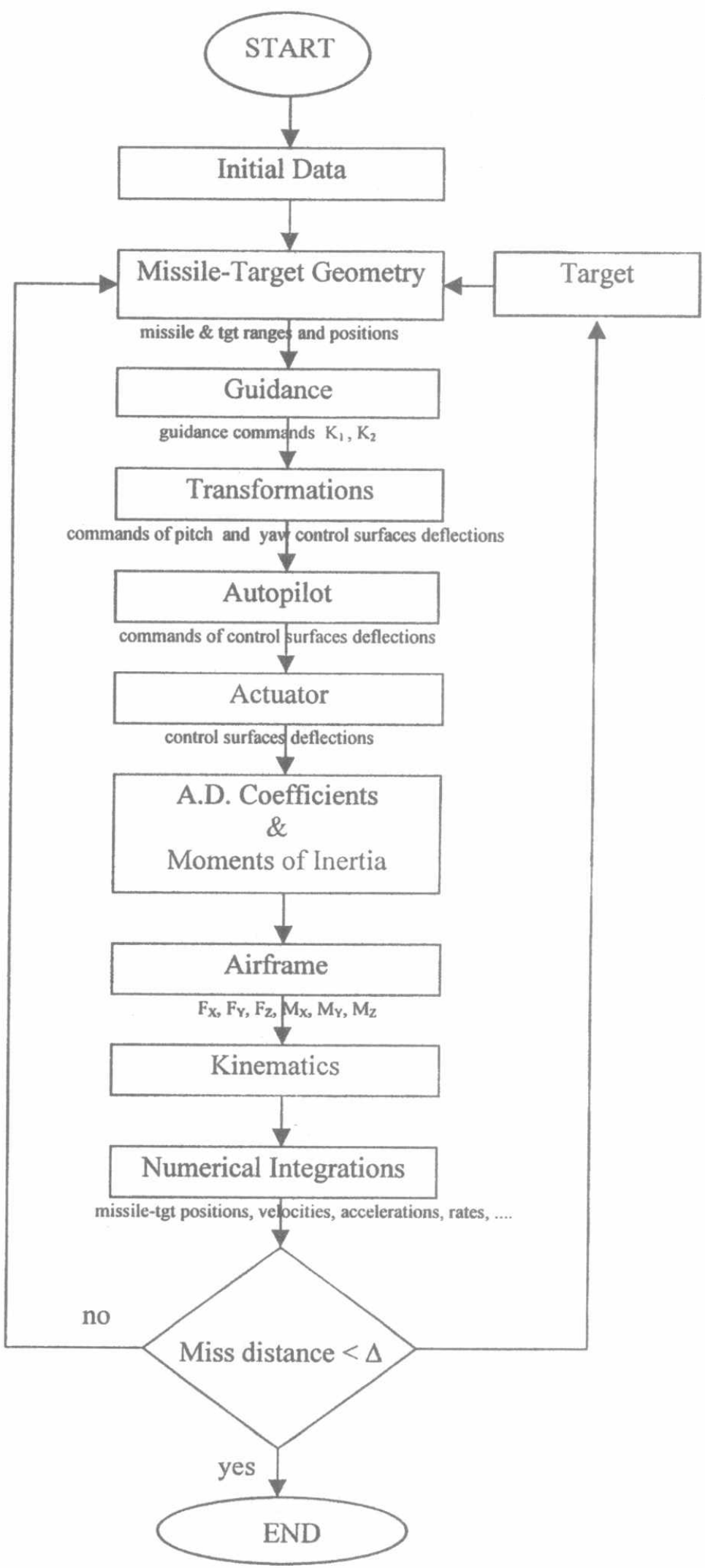

Fig. 10: 6 DOF missile-target simulation model 
Proceedings of the $2^{\text {nd }}$ ICEENG Conference, 23-25 Nov. 1999

\begin{tabular}{|l|l|}
\hline RG-9 & 14 \\
\hline
\end{tabular}

Consider a target flying in the space with the initial conditions: $\mathrm{x}_{\mathrm{t}}=10 \mathrm{~km}, \mathrm{y}_{\mathrm{t}}=9 \mathrm{~km}, \mathrm{z}_{\mathrm{t}}=5 \mathrm{~km}$, $\mathrm{vt}_{\mathrm{x}}=300 \mathrm{~m} / \mathrm{sec}$. Then, The flight path trajectories for the simplified and complete approaches are as shown in Figures 11, and 12 respectively [10] and [11].

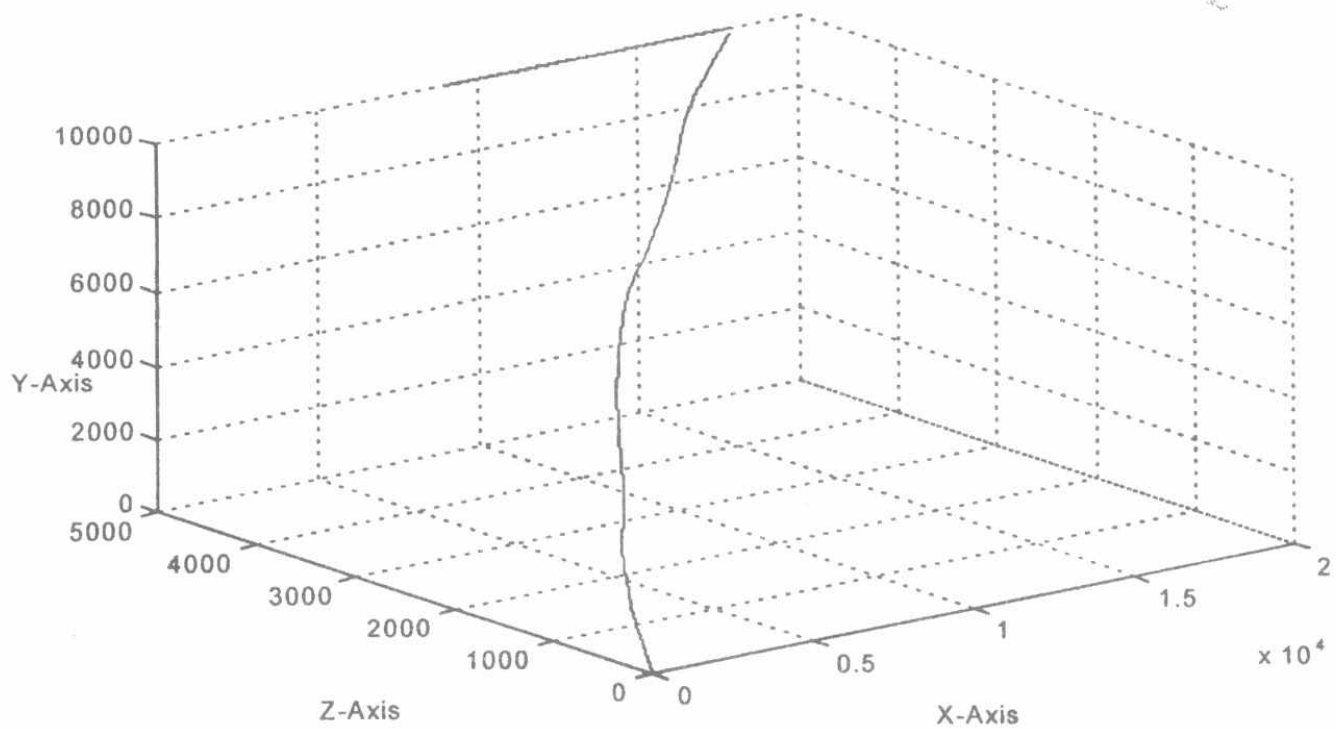

Fig. 11: Missile-target engagement for the simplified approach

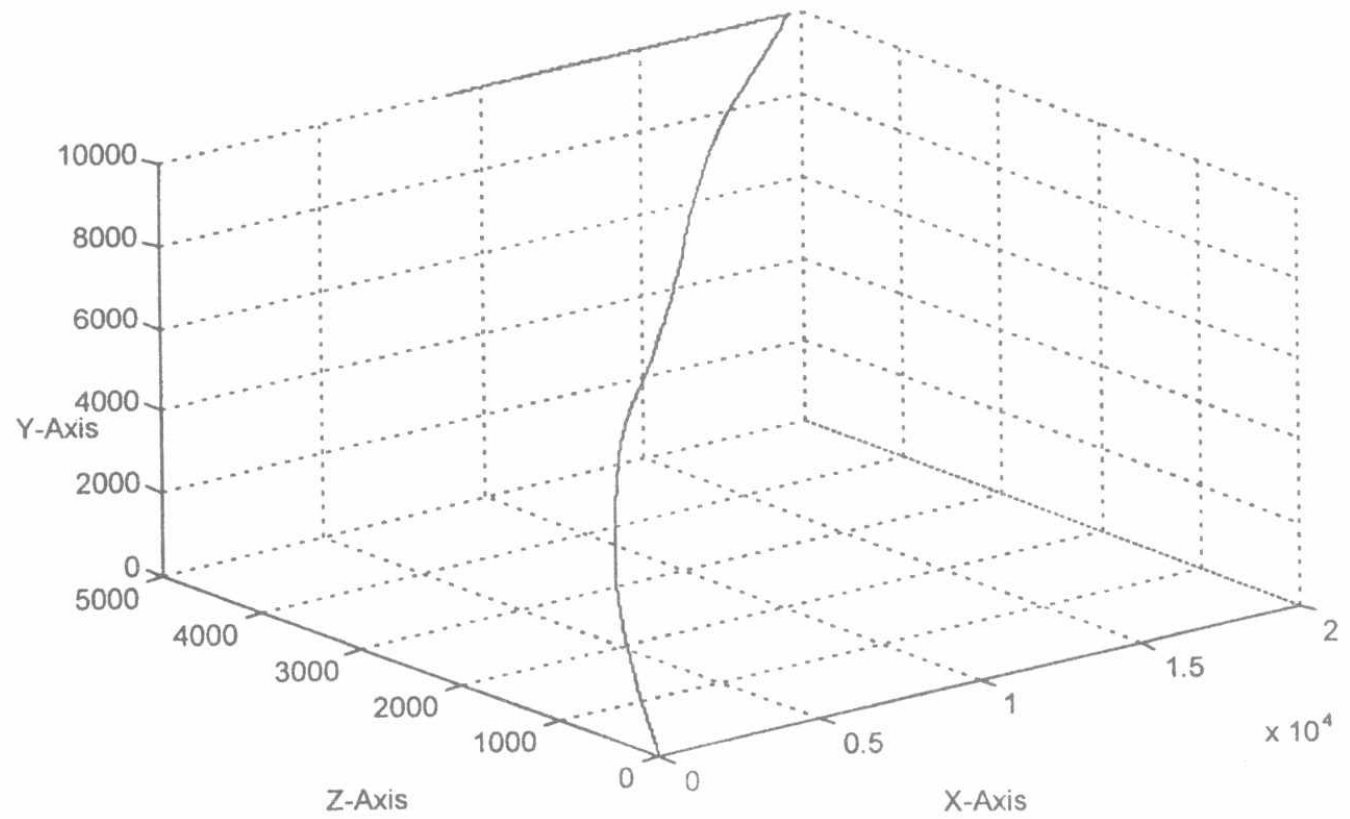

Fig. 12: Missile-target engagement for the complete approach

It is clear that when carefuly analyzing the aerodynamic performance for the first stage of the missile the final missile-target miss distance decreases considerabily. Table 1 shows the final 
Proceedings of the $2^{\text {nd }}$ ICEENG Conference, 23-25 Nov. 1999

\begin{tabular}{|l|l|}
\hline $\mathrm{RG}-9$ & 15 \\
\hline
\end{tabular}

miss distance, normal acceleration, and time of flight for the simplified and complete approaches.

Table 1: Final Flight characteristics for the simplified and complete approaches

\begin{tabular}{|l|c|c|c|}
\hline \multicolumn{5}{|c|}{ Target Parameters } \\
\hline & $\begin{array}{c}\text { miss distance } \\
(\mathbf{m})\end{array}$ & $\begin{array}{c}\text { normal acceleration } \\
\left(\mathbf{m} / \mathbf{s e c}^{\wedge} \mathbf{2}\right)\end{array}$ & $\begin{array}{c}\text { time of flight } \\
\text { (sec) }\end{array}$ \\
\hline Simplified approach & 442.47 & 36.72 & 29.93 \\
\hline Complete approach & 12.59 & 8.28 & 30.27 \\
\hline
\end{tabular}

The results show that the final miss distance and normal acceleration decrease considerably when accurately analyzing the aerodynamic performance of the first stage of the missile.

\section{4-CONCLUSIONS}

The aerodynamic performance of a missile depends on many parameters such as its configuration, dimensions, atmosphere, mach number and Reynolds numbers values, angle of attack, sideslip angle, control surfaces deflections and other flight parameters. The behavior of the aerodynamic characteristics of the missile varies considerably from the subsonic to the transonic to the supersonic ranges.

The complete approach for calculating the aerodynamic derivatives (over the whole range of mach number during the missile flight envelope) is a more realistic procedure and provides better flight results (small miss distance and small normal acceleration) than calculating these derivatives only in the super-sonic range depending on the simplified approach.

The mission of the control system (autopilot) is more active when using the complete approach than using the simplified approach (i.e. The correction of the displacement between the ideal and actual trajectories of the underlying missile is more active depending on the complete approach).

\section{REFERENCES:}

[1]Hemsch M. J. (Ed.), Tactical Missile Aerodynamics: General Topics, AIAA, Vol. 141, 1996.

[2]Cook, M.V., Flight Dynamics Principles, New York, 1996.

[3]Emil J. (Ed.), Test and Evaluation of The Tactical Missile, AIAA, 1994.

[4]Beer, P. F., and E.Russel, Vector Mechanics for Engineers Dynamics, New York, 1981.

[5] Lecture Notes, Anti-aircraft Guided Missile System, M.T.C., 1973.

[6]Pitts, W.C., and J.N. Kaatarari, Lift and Center of Pressure of Wing-Body-Tail Combination at Subsonic, Transonic, and Supersonic Speeds, M.T.C., NASA Report 1307.

[7]Chernobrobky, L., Dynamic Flight of Non Pilot Vehicle, Moscow, 1973.

[8]O.E. Abdelhamid, Lecture Notes in Aerodynamic, M.T.C., Cairo, 1998.

[9]Hemsch, M. J. (Ed.), Tactical Missile Aerodynamics, AIAA, Vol. 104, 1986.

[10]Macfadzean, R. H.M., Surface-Based Air Defense System Analysis, AIAA, 1993.

[11]Garnell P., and D.J.East, Guided Weapon Control Systems, Pergamon Press, New York, 1977. 
